\title{
Non-Planckian behaviour of burst spectra: dependence of the blackbody radius on the duration of bursts
}

\author{
E. Damen, ${ }^{1}$ F. Jansen, ${ }^{2}$ W. Penninx,,${ }^{1,3}$ \\ T. Oosterbroek, ${ }^{1}$ J. van Paradijs ${ }^{1}$ and W. H. G. Lewin ${ }^{3}$ \\ ${ }^{1}$ Astronomical Institute 'Anton Pannekoek', University of Amsterdam, Roetersstraat 15, \\ 1018 WB Amsterdam, The Netherlands \\ ${ }^{2}$ Space Research Laboratory, Postbus 9504, 2300 RA Leiden, The Netherlands \\ ${ }^{3}$ Center for Space Research and Department of Physics, Massachusetts Institute of \\ Technology 37-627, Cambridge, MA 02139, USA
}

Accepted 1988 October 2. Received 1988 October 1; in original form 1988 June 30

\begin{abstract}
Summary. From a statistical study of X-ray bursts observed with EXOSAT from $4 \mathrm{U} / \mathrm{MXB} 1636-53$ and $E X O 0748-67$, we find a strong correlation between the burst duration and the blackbody temperature, $k T_{\mathrm{bb}}$, as measured at the moment that the flux has decayed to 10 per cent of the Eddington flux. We find no correlation between the persistent flux near the burst and this temperature nor with the duration of the burst.

Since the burst duration probably depends on the chemical composition of the bursting layers, these results suggest that: (i) burst spectra show deviations from a Planckian spectrum, which depend on the chemical composition of the bursting layer, and (ii) this composition is not a simple function of the persistent flux and consequently of the accretion rate.

In view of this, it is not possible to convert observed colour temperatures into effective temperatures, which makes it difficult to determine reliable estimates of the neutron star radius.
\end{abstract}

\section{Introduction}

The spectra of X-ray bursts can be described reasonably well by a blackbody model. However, the emission from atmospheres, which are dominated by electron scattering (as is the case for neutron star surface layers during bursts), is expected to deviate substantially from that of a blackbody (see, e.g. van Paradijs 1982; Czerny \& Sztajno 1983). Detailed knowledge of these deviations, which can be expressed for example in the ratio of a colour temperature $T_{c}$ (determined from a blackbody fit to the spectrum) to the effective temperature. $T_{\text {eff }}$, is important in attempts to determine the mass-radius relation of neutron stars from burst observations (Sztajno et al. 1987; van Paradijs \& Lewin 1987, 1988). Several authors have published results of numerical models of radiative-equilibrium neutron-star atmospheres, 
taking into account inelastic electron scattering and free-free and bound-free absorption processes (London, Taam \& Howard 1984, 1986; Syunyaev \& Titarchuk 1986; Lapidus, Syunyaev \& Titarchuk 1986; Ebisuzaki \& Nomoto 1986; Foster, Ross \& Fabian 1986).

Observations of bursts from 4U/MXB 1636-53 and 4U 1608-52 (Matsuoka 1987) and EXO 0748-676 (Gottwald et al. 1986) have shown that bursts from a single source sometimes show different blackbody radii at the same colour temperature, e.g. in the case of 0748 - 676, Gottwald et al. (1986) found a decrease in the apparent blackbody radii during the burst decay by a factor of $\sim 2$ as the strength of the persistent emission decreased by a factor of $\sim 2$.

These results stimulated us to start an analysis in a uniform manner of all X-ray bursts observed by EXOSAT. This will make it possible to study a large number of burst spectra and will greatly improve the significance of the results. In this paper we report some results of the spectral analysis of all X-ray bursts observed with EXOSAT from 1636-53 and 0748-676, emphasizing the deviations from a blackbody. We confirm that the burst spectra for these sources are markedly different from those of blackbodies and that the blackbody radii for different bursts (at a fixed colour temperature) are not always the same. In particular, we find a strong correlation between the duration of the burst and the blackbody temperature as measured at a fixed flux level.

\section{Observations and method of analysis}

During its 3-yr lifetime, EXOSAT observed the galactic low-mass X-ray binaries 4U/MXB 1636-53 and EXO 0748-676 for approximately 180 and $114 \mathrm{hr}$, respectively. During these observations $60 \mathrm{X}$-ray bursts were recorded from 1636-53 and 35 from $0748-676$. One burst from 1636-53 was observed with a time resolution of $10 \mathrm{~s}$, which is not suitable for a detailed time-resolved spectral analysis, and is not included in our analysis. Two bursts from 0748 - 676 occurred during X-ray eclipse of the source, and have also been excluded from our analysis. Several bursts have been analysed and described before (1636-53: Turner \& Breedon 1984; Sztajno et al. 1985; Lewin et al. 1987; 0748-676: Gottwald et al. 1986, 1987). Using an adapted version of an EXOSAT data analysis package developed at the Space Research Laboratory in Leiden (ROL), we (re-)analysed all bursts in a uniform way.

In this analysis, spectra are accumulated over time intervals that vary between $\sim 1$ s near the peak, and $\sim 10-20 \mathrm{~s}$ during the decay of the burst. Before every burst a spectrum is accumulated (for $100 \mathrm{~s}$ ) of persistent emission plus background, and subtracted from the burst spectra. The average number of spectra per burst is 16 for $1636-53$, and 10 for $0748-676$. All spectra are fitted with a blackbody model which includes a correction for a low-energy cut-off, with a fixed value for the equivalent hydrogen column density $N_{\mathrm{H}}$. We used values of $5.6 \times 10^{21}$ and $0.0 \mathrm{~cm}^{-2}$ for the equivalent hydrogen column densities towards 1636-53 and 0748-676, respectively. These values have been derived from optical observations (Gorenstein 1975; Lawrence et al. 1983; Schmidtke \& Cowley 1987). The resulting fitted colour temperature, $k T_{c}$, is then used to calculate a photon spectrum at the source and a bolometric correction, thus yielding the bolometric flux at any point in time in the burst. For every burst the results are plotted in a $\log F_{\text {bol }}$ versus $\log k T_{\mathrm{c}}$ diagram, comparable to a Hertzsprung-Russell diagram (Ebisuzaki, Hanawa \& Sugimoto 1984; Sugimoto, Ebisuzaki \& Hanawa 1984).

To determine the flux in the persistent emission near every burst, we accumulated $1000 \mathrm{~s}$ of persistent emission (background subtracted) in a spectrum, which we fitted with a power-law spectral model with an exponential high-energy cut-off of the form $f(E)=E^{-\Gamma} \exp \left[\left(E_{\mathrm{c}}-E\right) / E_{f}\right]$. Here $E_{\mathrm{c}}$ is the energy at which the cut-off starts, and $E_{f}$ deter- 
mines the 'steepness' of the cut-off. In addition an interstellar absorption component with an equivalent hydrogen column density $N_{\mathrm{H}}$ is included. For spectra from $1636-53$, we fixed $\Gamma$ at a value of 2.0 and varied $N_{\mathrm{H}}, E_{\mathrm{c}}$ and $E_{f}$. For spectra from $0748-676$, we fixed $N_{\mathrm{H}}$ at a value of $0.0 \mathrm{~cm}^{-2}$ and varied $\Gamma, E_{\mathrm{c}}$ and $E_{f}$. These models gave acceptable fits in almost all cases for both 1636-53 and 0748-676. Since the fit parameters are strongly depending on each other, which results in a variable fitted value for $N_{\mathrm{H}}$ that is probably not realistic, we determined the persistent flux between 1.5 and $15.0 \mathrm{keV}$ as measured at the detector. As long as the absorption near the source (i.e. intrinsic to the source itself) is not varying and the fit is acceptable, the resulting value is an acceptable measure of the source flux. If we assume that the fitted values of $N_{\mathrm{H}}$ are real, the resulting flux, corrected for $N_{\mathrm{H}}$, is larger by a factor varying between 5 and 15 per cent, depending on flux level. However, this difference will not affect our conclusions.

\section{Results}

Fig. 1 gives three representative bursts in one Hertzsprung-Russell diagram for each source. Data points from the rising parts of the burst were excluded from this diagram, since these points give large uncertainties in the colour temperature. Typical errors for several flux levels are indicated on the left part of the diagram. These diagrams clearly show that the cooling tracks differ from burst to burst, and that bursts do not exactly behave like a cooling blackbody with a fixed radius (the latter would have a cooling track with a slope of 4 , as indicated by the
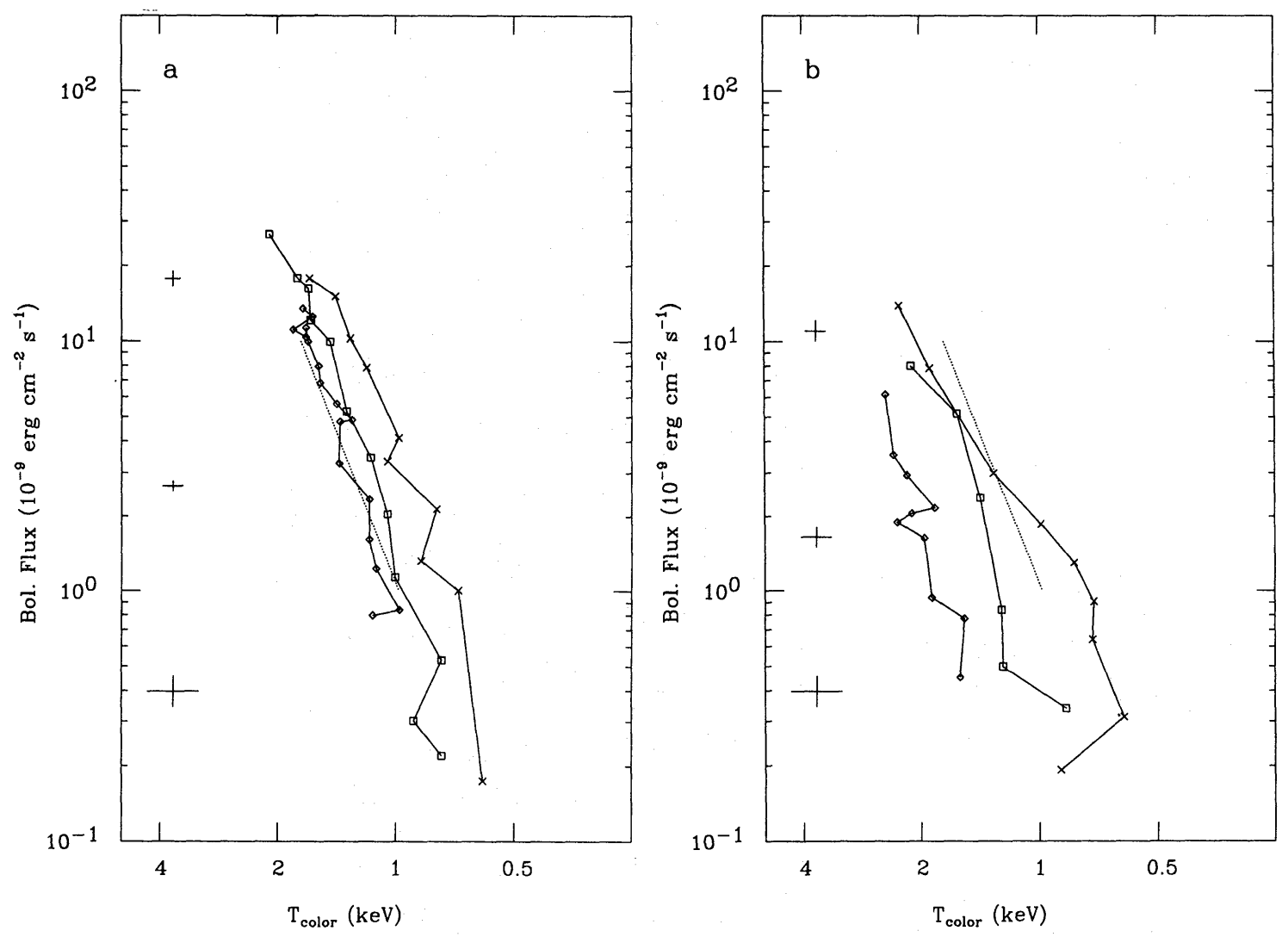

Figure 1. Diagram of bolometric flux versus fitted colour temperature of three different bursts from 1636-53 (a) and $0748-676$ (b). Clearly, the position and slope of cooling tracks can differ for bursts from a single source. The dotted line in both diagrams is a theoretical cooling track for a blackbody with a radius of $10 \mathrm{~km}$ at a distance of $10 \mathrm{kpc}$. Typical $1 \sigma$ errors at several flux levels are indicted in the left part of the diagrams. 
dotted line). The difference in temperature at the same flux level is equivalent to a difference in blackbody radius, according to the relation:

$4 \pi d^{2} F_{\mathrm{bol}}=4 \pi R_{\mathrm{bb}}^{2} \sigma T_{\mathrm{c}}^{4}$.

Here $d$ is the distance to the source, $F_{\mathrm{bol}}$ is the observed bolometric flux, $R_{\mathrm{bb}}$ is the observed blackbody radius and $T_{\mathrm{c}}$ is the observed fitted colour temperature.

To analyse the differences between bursts in a more quantitative way, we made linear leastsquare fits to the data points in the HR-diagram for each burst separately. In the case of $1636-53$ we limited this fit to the flux range $0.05 F_{\text {edd }}$ to $0.3 F_{\text {edd }}$; here the Eddington flux, $F_{\text {edd }}$ $\left(=6.3 \times 10^{-8} \mathrm{erg} \mathrm{cm}^{-2} \mathrm{~s}^{-1}\right)$, equals the observed average maximum flux of bursts clearly showing radius expansion. This part of the cooling track can be approximated by a straight line in the HR-diagram for all bursts. In the case of $0748-676$ we extended the range of the fit to $0.02 F_{\text {edd }}$ (here $F_{\text {edd }}=4.0 \times 10^{-8} \mathrm{erg} \mathrm{cm}^{-2} \mathrm{~s}^{-1}$, as observed for bursts clearly showing radius expansion), because the bursts from this source are weak compared to the persistent emission, resulting in too few data points in the original fitting range. For both sources, the fit is centred at the 10 per cent Eddington flux level.

There are two effects which could affect the results systematically:

(i) Using such small fractions of the Eddington flux for the fits could give rise to systematic errors if the persistent flux is not independent of the burst flux, e.g. when the persistent flux contains a blackbody (or blackbody-like) contribution from the same neutron-star atmosphere that transports the burst emission. However, even when all persistent flux is in this blackbody, the contribution of this flux is about as large as the lower flux limit of the fit in 1636-53 and only about 25 per cent of the lower flux limit of the fit in $0748-676$. In the case of 1636-53 this could give serious effects on the fit results. Two component fits on spectra of the persistent emission from 1636-53, using various combinations of a blackbody and some other model (power law, thermal bremsstrahlung and power law plus high-energy cut-off), show that at most $\sim 15$ per cent of the total persistent emission is emitted in a low temperature blackbody $\left(k T_{\mathrm{bb}}<0.5 \mathrm{keV}\right)$. This small fraction will not affect general trends in the fit results.

(ii) In principle, the fitted colour temperature of a spectrum may be systematically affected by long accumulation times of the spectrum, due to smearing of spectra at different flux levels (and temperatures). However, within the fitting range, the accumulation times of the spectra vary between $\sim 2 \mathrm{~s}$ at the $0.3 F_{\text {edd }}$ level and $\sim 5 \mathrm{~s}$ at the $0.05 F_{\text {edd }}$ level, with a mean of $2.2 \pm 0.9 \mathrm{~s}$ at the $0.1 F_{\text {edd }}$ level. Compared to the flux and temperature changes in the burst, these accumulation times are short enough for this systematic effect to be unimportant.

The results are expressed in the two fit parameters: the slope of the fit, $\Sigma$, and the value of $k T_{\mathrm{c}}$ at 10 per cent of the Eddington flux, $k T_{0.1}$.

We searched for possible correlations of the fit parameters with other burst properties, and found that the duration of the bursts $\tau$ (defined as the ratio of total burst fluence and peak flux, $\left.E_{\mathrm{b}} / F_{\max }\right)$ is correlated with $k T_{0.1}$. This correlation is displayed in Fig. 2(a) and (b) for 1636-53 and $0748-676$, respectively. The correlation is very strong in the case of $1636-53$, and substantially weaker (but definitely present) in $0748-676$. The point designated with ' $T$ ' in Fig. 2(a) is the triple burst (van Paradijs et al. 1986; Penninx, van Paradijs \& Lewin 1987), which shows three peaks in its bolometric flux profile, probably caused by multiple release of energy. Because of this multiple release, the burst has a relatively long duration but a 'normal' cooling track, shifting it to the right in the diagram.

As mentioned above, a higher value for $k T_{0.1}$ corresponds to a lower blackbody radius. Our findings confirm the results by Matsuoko (1987) and Gottwald et al. (1986, 1987), but they did not relate the differences in radii to the duration of the bursts. 

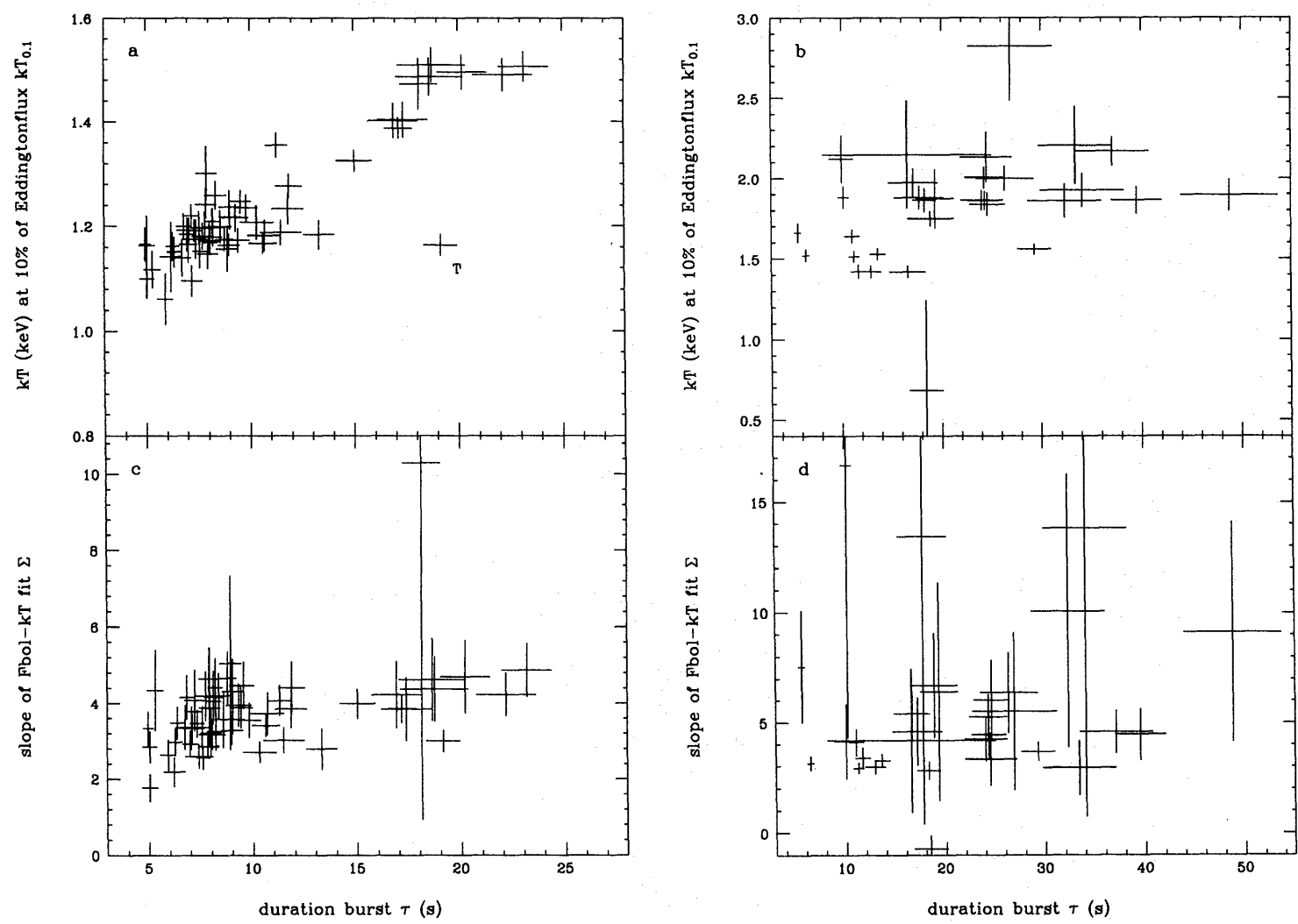

Figure 2. (a) and (b): relation between the duration of bursts and the fitted colour temperature at the moment that the flux has decayed to 10 per cent of the Eddington flux, for $1636-53$ (a) and $0748-676$ (b); (c) and (d): relation between the duration of the bursts and the slope of the cooling tracks in the HR-diagram for 1636-53 (c) and 0748-676 (d). The data point designated ' $T$ ' in (a) is the triple burst. Errors are $1 \sigma$ formal errors. See text for more details.

Fig. 2(c) and (d) gives the fitted slope of the cooling track, $\Sigma$, against the duration of the bursts $\tau$ for $1636-53$ and $0748-676$, respectively. For $1636-53$, there is a correlation between these two parameters which seems to level out for $\tau \gtrsim 10 \mathrm{~s}$. The average value of the slope increases from $\sim 2.5$ for bursts with $\tau \leqslant 6 \mathrm{~s}$ to $\sim 4$ for bursts with $\tau \gtrsim 15 \mathrm{~s}$. The latter value $(\Sigma=4)$ equals the value expected for Planckian spectra. In the case of $0748-676$, many data points show large error bars, due to the fact that most of the fits in the HR-diagram are done on only very few points (but always more than two). Fig. 2(d) shows no correlation between $\Sigma$ and $\tau$ for $0748-676$, as in 1636-53. The weighted mean of $\Sigma$ for all data points is $3.2 \pm 0.1$, which becomes $3.3 \pm 0.1$ if we exclude the single data point with a negative value. The latter value is consistent with a constant slope for all cooling tracks of this source.

\section{Discussion and conclusions}

We find that the fitted colour temperature at the 10 per cent Eddington flux level in the burst, $k T_{0.1}$, is correlated with the burst duration $\tau$ and that the slope $\Sigma$ of the cooling track of bursts in the HR-diagram, in the case of $1636-53$, is smaller for short bursts than for long bursts. In principle there could be several possible explanations for this: (i) variable anisotropy of the burst radiation; (ii) variation in the size of the radiating area, and (iii) non-Planckian behaviour of the burst spectra. We will discuss each of these possibilities separately. 
(i) Variable anisotropy of burst. Since the systems considered here are not spherically symmetrical, it is likely that the emitted burst radiation is not isotropic (Lapidus \& Syunyaev 1985; Fujimoto 1988). However, the question of relevance here is whether the anisotropy is constant throughout a burst and has the same value in every burst from a given source. In principle a variable anisotropy can explain both the shift in position of the cooling tracks of different bursts in the HR-diagram (i.e. the variation in $k T_{0.1}$ ) and the changes in the slope of the cooling tracks. A shift in position will arise from a variable anisotropy from burst to burst in the sense that a higher anisotropy factor shifts the cooling track of the burst to higher temperatures (to the left in the HR-diagram). A change in slope will arise from a changing anisotropy factor during the burst in the sense that the slope will be steeper when the anisotropy factor increases during the burst. To explain the observed shifts and slope changes of cooling tracks of bursts from 1636-53, we need a change in anisotropy by a factor $\sim 4$ between bursts and by a factor $\sim 3$ during bursts. For 0748 -676 these factors are $\sim 20$ and $\sim 2$, respectively. These large changes in anisotropy are very unlikely, considering the fact that bursts from 1636-53 show a well defined linear relation between peak flux of the burst (not showing radius expansion) and the waiting time since the previous burst, with a scatter of $\sim 30$ per cent around the best fitting relation (Lewin et al. 1987). This scatter would be $\sim 200$ per cent, if the anisotropy indeed changed by the above factor. In addition, bursts from this source show a gap in their peak flux distribution, which can be explained by a difference in Eddington flux for hydrogen-rich atmospheres and hydrogen-poor atmospheres (Sugimoto $e t$ al. 1984). This gap would disappear if the anisotropy changed by a factor of $\sim 1.3$ or more between bursts, a value much lower than that needed to explain our observations in terms of a variable anisotropy. Moreover, quite independently, it is difficult to explain the observed relation between $k T_{0.1}$ and $\tau$ with a changing anisotropy. This could perhaps be done if we assume the formation of an accretion disc corona during the burst, which increases the anisotropy and delays photons from the bursts due to (compton) scattering (Melia 1987; however see also Penninx et al. 1987). However, one would expect the formation of such a burst-induced corona only during the most energetic bursts, after a relatively long waiting time ( $\gtrsim 5 \mathrm{hr}$ ). Our observations show that for both sources, the bursts with the longest duration are by no means the most energetic and occur after a waiting time of only $\sim 1 \mathrm{hr}$. We thus conclude that it is very unlikely that a variable anisotropy causes the observed differences in the cooling tracks of bursts from $1636-53$ and $0748-676$.

(ii) Variable radiating area. A radiating area which changes from burst to burst, and during the bursts, could also explain the observed differences in cooling tracks or bursts. However, the same arguments concerning the distribution of peak fluxes, mentioned above, can be used against this possibility. In addition, to explain our observations there should be an anti-correlation between the duration of the bursts and the radiating area. There is no obvious reason why this would be the case. We therefore conclude that it is unlikely that a variable radiating area causes the observed differences in cooling tracks.

(iii) Non-Planckian behaviour of spectra. The observed (fitted) colour temperature, $T_{c}$, of a burst spectrum is not equal to the effective temperature $T_{\text {eff }}$, due to deviations from a pure Planck curve. Theoretical models predict that the ratio $T_{\mathrm{c}} / T_{\text {eff }}$ is a function of $T_{\text {eff }}$ (Czerny \& Sztajno 1983; van Paradijs 1982; London et al. 1984, 1986; Ebisuzaki \& Nomoto 1986; Foster et al. 1986; Lapidus et al. 1986; Syunyaev \& Titarchuk 1986; Babul \& Paczynski 1987), which can explain, in principle, both the shifts in position and the slope changes of the cooling tracks in the HR-diagram. In the case of 0748-676 all bursts, and for 1636-53 those bursts with $\tau \gtrsim 15 \mathrm{~s}$, show almost no differences in the slope of the cooling tracks (in 1636-53 this constant slope is $\sim 4$, in $0748-676$ this slope is $\sim 3.3$; see also Section 3 ). This could mean that for those bursts $T_{\mathrm{c}} / T_{\text {eff }}$ has a constant value which, however, differs from burst to 
burst to explain the differences in position of the cooling tracks. This seems rather contrived (however, see also the discussion on chemical composition, below). If we assume that for the burst with the lowest $k T_{0.1}$ (the one at the far right in the HR-diagram) $T_{\mathrm{c}} / T_{\text {eff }}=1$ (which, admittedly, is rather arbitrary; however, see below), we can give upper limits on the value of $T_{\mathrm{c}} /$ $T_{\text {eff }}$, needed to explain the shifts of the cooling tracks in the HR-diagram. These upper limits are $T_{\mathrm{c}} / T_{\text {eff }} \sim 1.4$ and $\sim 2$ for $1636-53$ and $0748-676$, respectively. Theoretical models predict these kind of values for $T_{c} / T_{\text {eff }}$. However, note that we assumed that the burst with the lowest $k T_{0.1}$ has $T_{\mathrm{c}} / T_{\text {eff }}=1$. It is likely that this value is larger than one (at present, no model predicts values of $T_{\mathrm{c}} / T_{\text {eff }}<1$ ), and this increases the upper limits.

There is evidence that the burst duration depends on the chemical composition of the flashing layer (see e.g. Fujimoto, Hanawa \& Miyaji 1981; van Paradijs, Penninx \& Lewin 1988, and references therein). Bursts which are due to helium flashes in a hydrogen-rich environment last longer than pure helium bursts due to the relatively long time-scales involved in proton capture processes. The shift of position in the HR-diagram of the cooling tracks of long bursts, relative to those of short bursts, is in qualitative agreement with theoretical cooling tracks of hydrogen-rich and hydrogen-poor bursts (Ebisuzaki \& Nakamura 1988). However, the shifts in position that we observe are much larger than predicted by these models. Our result can be explained qualitatively if the burst spectra are non-Planckian, and if they are related to the chemical composition of the flashing layer. There could, for instance, be a mixing between material of the flashing layer and the neutron-star atmosphere during bursts. Our results would then indicate that the composition of the neutron star atmosphere is an important parameter which has to be taken into account in the determination of the mass-radius relation of neutron stars (van Paradijs \& Lewin 1987).

Burst models of Fujimoto, Hanawa \& Miyaji (1981) indicate that the chemical composition of the bursting layer is determined by the mass accretion rate. Both at very high and very low accretion rates, the flashing layer is expected to be hydrogen rich. One could thus expect a dependence of burst duration on the persistent flux of the source (assuming that the persistent flux is a direct measure of the accretion rate). Van Paradijs, Penninx \& Lewin (1988) present a diagram of $\log \tau$ versus $\log \gamma$ (where $\gamma$ is the ratio of persistent flux to Eddington flux) for several sources, which is in good qualitative agreement with the model of Fujimoto et al. (1981).

Fig. 3(a) gives a diagram of burst duration versus persistent flux near the burst for 1636-53. There seems to be no correlation between duration and persistent flux, however, two different states are visible. At the same persistent flux level, bursts with a long duration (all from the 1983 observing run) and with a short duration (from the 1984 and 1985 observing runs) have been observed. There appears to be a gap in the distribution of $\tau$. This diagram is not in contradiction with the $\log \tau$ versus $\log \gamma$ diagram of van Paradijs, Penninx \& Lewin (1988). Due to the small range of persistent flux in 1636-53, our results fall completely within the scatter of their diagram. Based on this comparison, we conclude that on a large scale (persistent flux changes by a factor $\sim 50$ or more) the duration of bursts (and thus the chemical composition of the flashing layer) is determined by the accretion rate, but that on a small scale additional parameters are involved in determining $\tau$.

Fig. 3(b) gives the equivalent diagram for $0748-676$. Here we see only one state and again no correlation between $\tau$ and the persistent flux. However, there seems to be an upper limit on $\tau$ which is a function of persistent flux. This result qualitatively confirms the result by Gottwald et al. (1987). However, we find no anti-correlation between burst duration and persistent flux, as reported by these authors using the same data. This different conclusion can be caused by source variability: Gottwald et al. (1987) accumulated their persistent spectra over much 

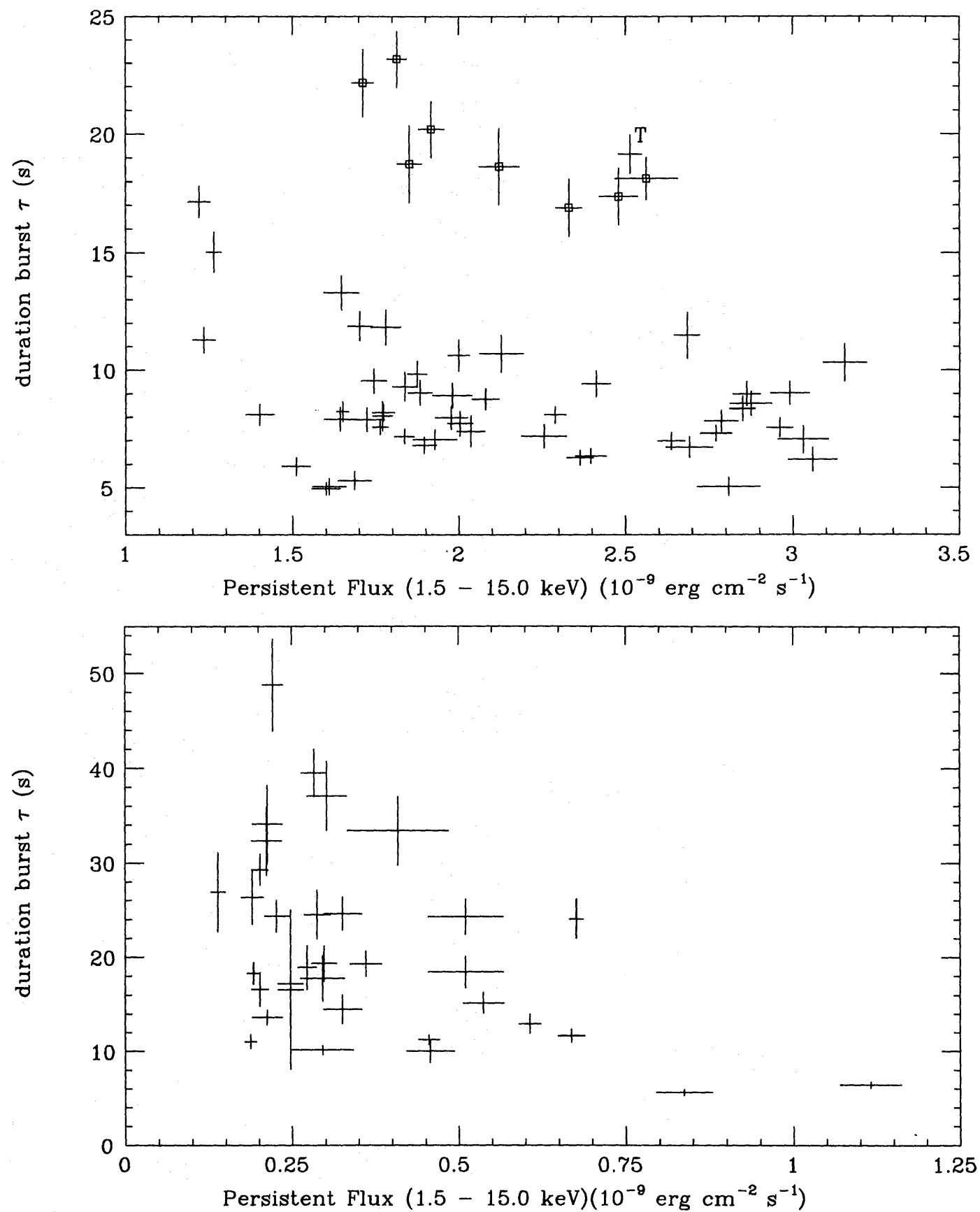

Figure 3. Relation between the persistent flux near the burst as measured at the detector and the duration of the burst for $1636-53$ (a) and $0748-676$ (b). Squares in (a) are data points from bursts observed in the 1983 observing run, all other data points in (a) are from 1984 and 1985 observations. The data point designated ' $T$ ' in $(a)$ is the triple burst. Errors are $1 \sigma$ formal errors.

longer time intervals than we did. If we compare Fig. 3(b) with the $\log \tau$ versus $\log \gamma$ diagram of van Paradijs et al. (1988) we conclude that our results fit in their diagram reasonably well. Our data points at low persistent flux are at lower $\tau$-values than expected from their diagram. Again, this can be explained by the difference in accumulation time of the persistent flux.

Summarizing we conclude:

(i) The observed changes in position and slope of cooling tracks of bursts from a single source in the HR-diagram are most likely caused by the non-Planckian behaviour of the burst spectra and by differences in the chemical composition of the bursting layer. 
(ii) Differences in chemical composition of the bursting layer can account for the observed correlation between the duration of bursts, $\tau$, and the position of the cooling track in the HRdiagram.

(iii) The level of persistent flux (and thus the accretion rate) determines the chemical composition of the bursting layer (and thus the duration of bursts) on a large scale (persistent flux changes by a factor $\sim 50$ or more). On a small scale additional parameters are involved.

\section{Acknowledgments}

ED thanks the directors of the Space Research Laboratory Leiden (ROL) and the Max-Planck Institut für Extraterrestrische Physik (MPE) in garching bei München for their hospitality and help during the initial stages of this research. ED is supported by the Netherlands Organization for the Advancement of Research NWO/ASTRON under contract No. 782371-019. WHGL acknowledges support from the US National Aeronautics and Space Administration under grants NAG8-571, NAG8-674 and NSG-7643.

\section{References}

Babul, A. \& Paczynski, B., 1987. Astrophys. J., 323, 582.

Czerny, M. \& Sztajno, M., 1983. Acta astr., 33, 213.

Ebisuzaki, T., Hanawa, T. \& Sugimoto, D., 1984. Publs astr. Soc. Japan, 36, 551.

Ebisuzaki, T. \& Nakamura, N., 1988. Astrophys. J., 328, 251.

Ebisuzaki, T. \& Nomoto, K., 1986. Astrophys. J., 305, L67.

Foster, A. J., Ross, R. R. \& Fabian, A. C., 1986. Mon. Not. R. astr. Soc., 221, 409.

Fujimoto, M. Y., 1988. Astrophys. J., 324, 995.

Fujimoto, M. Y., Hanawa, T. \& Miyaji, S., 1981. Astrophys. J., 247, 267.

Gorenstein, P., 1975. Astrophys. J., 198, 95.

Gottwald, M., Haberl, F., Parmar, A. N. \& White N., 1986. Astrophys. J., 308, 213.

Gottwald, M., Haberl, F., Parmar, A. N. \& White N., 1987. Astrophys. J., 323, 575.

Lapidus, I. I. \& Syunyaev, R. A., 1985. Mon. Not. R. astr. Soc., 217, 291.

Lapidus, I. I., Syunyaev, R. A. \& Titarchuk, L. G., 1986. Soviet Astr. Lett., 12, 383.

Lawrence, A. et al., 1983. Astrophys. J., 271, 793.

Lewin, W. H. G., Penninx, W., van Paradijs, J., Damen, E., Sztajno, M., Trümper, J. \& van der Klis, M., 1987. Astrophys. J., 319, 893.

London, R. A., Taam, R. E. \& Howard, W. M., 1984. Astrophys. J., 287, L27.

London, R. A., Taam, R. E. \& Howard, W. M., 1986. Astrophys. J., 306, 170.

Matsuoka, M., 1987. In: The Evolution of Galactic X-ray Binaries, Proc. NATO ASI, Rottach-Egern, FRG, 1985, p. 301, eds Trümper, J., Lewin, W. H. G. \& Brinkmann, W.

Melia, F., 1987. Astrophys. J., 315, L43.

Penninx, W., van Paradijs, J. \& Lewin, W. H. G., 1987. Astrophys. J., 321, L67.

Schmidtke, P. C. \& Cowley, A. P., 1987. Astr. J., 92, 374.

Sugimoto, D., Ebisuzaki, T. \& Hanawa, T., 1984. Publs astr. Soc. Japan, 36, 839.

Syunyaev, R., Titarchuk, L., 1986. Soviet Astr. Lett., 12, 359.

Sztajno, M., van Paradijs, J., Lewin, W. H. G., Trümper, J., Stollman, G., Pietsch, W. \& van der Klis, M., 1985. Astrophys. J., 299, 487.

Sztajno, M., Fujimoto, M. Y., van Paradijs, J., Vacca, W. D., Lewin, W. H. G., Penninx, W. \& Trümper, J., 1987. Mon. Not. R. astr. Soc., 226, 39.

Turner, M. \& Breedon, L., 1984. Mon. Not. R. astr. Soc., 208, 29P.

van Paradijs, J., 1982. Astr. Astrophys., 107, 51.

van Paradijs, J., Sztajno, M., Lewin, W. H. G., Trümper, J., Vacca, W. D. \& van der Klis, M., 1986. Mon. Not. R. astr. Soc., 221, 617.

van Paradijs, J. \& Lewin, W. H. G., 1987. Astr. Astrophys., 172, L20.

van Paradijs, J. \& Lewin, W. H. G., 1988. In: The Physics of Compact Objects: Theory versus Observations, Proc. COSPAR/IAU Symp., Sofia, Bulgaria, 1987, eds White, N. \& Filipov, L., Pergamon Press, in press.

van Paradijs, J., Penninx, W. \& Lewin, W. H. G., 1988. Mon. Not. R. astr. Soc., 233, 437. 